\title{
IMAGE CODING THROUGH Z- TRANSFORM WITH LOW ENERGY AND BANDWIDTH (IZEB)
}

\author{
Madhumita Sengupta, J. K. Mandal \\ Department of Computer Science and Engineering \\ University of Kalyani \\ Kalyani, Nadia, Pin. 741235, West Bengal, India \\ madhumita.sngptegmail.com, jkm.cse@gmail.com
}

\begin{abstract}
In this paper a Z-transform based image coding technique has been proposed. The techniques uses energy efficient and low bandwidth based invisible data embedding with a minimal computational complexity. In this technique near about half the bandwidth is required compared to the traditional Z-transform while transmitting the multimedia content such as images through network.
\end{abstract}

\section{KEYWORDS}

Z-Transform; Frequency Domain; Mean Square Error (MSE); Peak Signal to Noise Ratio (PSNR); Image Fidelity (IF).

\section{INTRODUCTION}

In today's digital world there is an enormous increase in the amount of multimedia content over internet such as image, video and audio materials. Such materials are traverse through wire and unwired medium in a carryon fashion.

Small digital device such as tablet, capsule, mobile, PDA's and many others are not yet small in physical senses due to their processing power and memory capacity. But the problem of sharing large amount of multimedia contents over internet creates a concern among researchers regarding the bandwidth utilization. In case of static spectrum assignment for mobile or radio networks bandwidth becomes a major concern.

Digital data are organized in a structure, traverse through network in a bit form of spatial data or frequency components. A single flip of bit is enough to destroy single pixel, but in case of frequency domain signal/images are first converted from spatial domain which decreases the probability of pixel value changes. 
Many transformation techniques such as DFT[1], DCT[2], DWT[3] and others are already implemented in digital world and widely used in today's technique such as steganography[4], data compression and many more.

This paper proposed a frequency domain based technique where the digital content such as image is converted form spatial domain to Z-domain using a $2 \times 2$ sliding window based mask in a row major to generate $2 \times 2$ real and $2 \times 2$ imaginary matrices through less amount of computation and without the trigonometric complexity. This paper also emphasis on transmitting the image over network where half of the $\mathrm{Z}$ domain coefficients are sufficient to regenerate the original image.

Various parametric tests are performed based on the original and reconstructed image at destination which shows that both the images are identical in terms of MSE as zero, PSNR as infinity and that of image fidelity as one.

Section 2 of this paper deals with the proposed technique through five sub sections. Results and discussions are outlined in section 3, conclusions are drawn in section 4 and references are given at end.

\section{THE TECHNIQUE}

The IZEB technique is divided into three major tasks. Forward Z-transform as describe in section 2.1, inverse Z-Transform given in section 2.2, and that of section 2.5 elaborate the procedure of bandwidth minimization. Traditional and fast Z-transform calculations are also given with example in section 2.3 and 2.4 respectively.

\subsection{Forward Z-Transformation}

Z-transform in signal processing converts a discrete time domain signal which is a sequence of real or complex numbers into a complex frequency domain representation. Z-transform can be defined in two ways, unilaterally or bilaterally.

In bilateral or two sided Z-transform of discrete time signal $\mathrm{x}[\mathrm{n}]$ is the formal power series $\mathrm{X}(\mathrm{z})$ defined by eq(1).

$$
X(\mathrm{z})=\mathrm{Z}\{\mathrm{x}[\mathrm{n}]\}=\sum_{\mathrm{n}=-\infty}^{\infty} \mathrm{x}[\mathrm{n}] \mathrm{z}^{-\mathrm{n}}
$$

Where $\mathrm{n}$ is an integer and $\mathrm{z}$ is, in general, a complex number.

Alternatively, in cases where $\mathrm{x}[\mathrm{n}]$ is defined only for $\mathrm{n} \geq 0$, the single sided or unilateral Ztransform is defined by eq(2).

$$
\begin{aligned}
& X(\mathrm{z})=\mathrm{Z}\{\mathrm{x}[\mathrm{n}]\}=\sum_{\mathrm{n}=0}^{\infty} \mathrm{x}[\mathrm{n}] \mathrm{z}^{-\mathrm{n}} \\
& \mathrm{z}=r e^{j \omega}=r(\cos \omega+j \sin \omega)
\end{aligned}
$$

Where $r$ is the magnitude of $z, j$ is the imaginary unit, and $\omega$ is the angle in radians. We get eq(3) by substituting the value of $z$ in eq(2). 


$$
\begin{aligned}
& X(\mathrm{z})=\mathrm{Z}\{\mathrm{x}[\mathrm{n}]\}=\sum_{\mathrm{n}=0}^{\infty} \mathrm{x}[\mathrm{n}] \mathrm{r}^{-\mathrm{n}} \mathrm{e}^{-\mathrm{j} \omega \mathrm{n}} \\
& \text { or } \quad X(\mathrm{z})=\mathrm{Z}\{\mathrm{x}[\mathrm{n}]\}=\sum_{\mathrm{n}=0}^{\infty} \mathrm{x}[\mathrm{n}] \mathrm{r}^{-\mathrm{n}}(\cos \omega+\mathrm{j} \sin \omega)^{-\mathrm{n}}
\end{aligned}
$$

On applying eq (3) for forward transformation on $2 \times 2$ mask of cover image in a row major order, four frequency component generates such as lower, horizontal, vertical and complex conjugate pair of horizontal frequency as shown in figure 1.a which is similar to subband coding[5]. Every frequency coefficient in lower to higher frequency bands are complex number in the format of ' $a$ $+j \mathrm{~b}$ '. Separation of real and imaginary parts is shown in figure 1.b and 1.c respectively.

\begin{tabular}{|c|c|}
\hline $\begin{array}{c}\text { Lower } \\
\text { Frequency } \\
(\text { LF })\end{array}$ & $\begin{array}{c}\text { Horizontal } \\
\text { frequency } \\
(H F)\end{array}$ \\
\hline $\begin{array}{c}\text { Vertical } \\
\text { frequency } \\
(V F)\end{array}$ & $\begin{array}{c}\text { Complex } \\
\text { conjugate } \\
\text { pair of } \\
(H F)\end{array}$ \\
\hline
\end{tabular}

(a) Z-coefficient quadrants of complex value ' $a+j$ '

\begin{tabular}{|c|c|}
\hline $\begin{array}{c}\text { Real part } \\
\text { of } L F\end{array}$ & $\begin{array}{c}\text { Real part } \\
\text { of } H F\end{array}$ \\
\hline $\begin{array}{c}\text { Real part } \\
\text { of } V F\end{array}$ & $\begin{array}{c}\text { Real part } \\
\text { of } H F\end{array}$ \\
\hline
\end{tabular}

(b) Real part of all frequency component ' $a$ '

\begin{tabular}{|c|l|}
\hline $\begin{array}{l}\text { Imaginary } \\
\text { part of LF }\end{array}$ & $\begin{array}{l}\text { Imaginary } \\
\text { part of } H F\end{array}$ \\
\hline $\begin{array}{l}\text { Imaginary } \\
\text { part of } V F\end{array}$ & $\begin{array}{l}\text { Negation of } \\
\text { Imaginary } \\
\text { part of } H F\end{array}$ \\
\hline
\end{tabular}

(c) Imaginary part of all frequency component ' $j \mathrm{~b}$ '

Fig. 1. Structural representation of forward Z-Transform (FZT) coefficients

\subsection{Inverse Z-Transformation}

Every transform technique exists with pair of equations, forward and inverse. The inverse Ztransform can be obtained by eq(4).

$$
x[n]=Z^{-1}\{X(z)\}=\frac{1}{2 \pi j} \oint_{C} X(z) z^{n-1} d z
$$

where $\mathrm{C}$ is a counter clockwise closed path encircling the origin and entirely in the region of convergence (ROC). A special case of this contour integral occurs when $\mathrm{C}$ is the unit circle. The inverse Z-transform simplifies to eq (5).

$$
x[n]=\frac{1}{2 \pi} \int_{-\pi}^{+\pi} X\left(e^{j \omega}\right) e^{j \omega n} d \omega
$$

The original gray scale image as shown in figure 2.a 'Map.pgm' on forward Z transform (FZT) generates four real and four imaginary subband as shown in figure 2.b and 2.d respectively. The information on the bands are emphasis through threshold as shown in figure 2.c and 2.e for real and imaginary parts respectively. Threshold increases the brightness of the small information present in the band. Inverse $\mathrm{Z}$ transform applied on real and imaginary parts generate lossless image back with a MSE[7] as zero and that of PSNR[7] is infinity. 


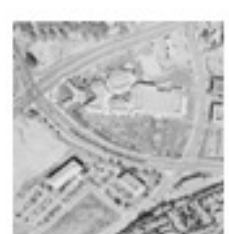

(a) Original Map

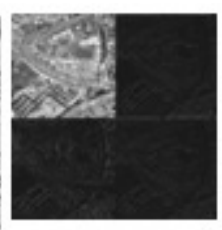

(b) Real value after FZT

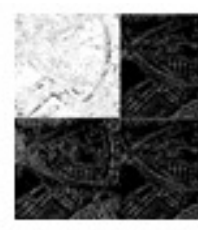

(c) Threshold Real valued part

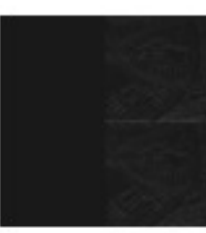

(d) Imaginary value after FZT

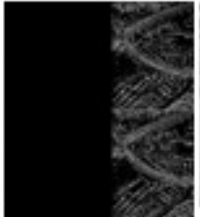

(e) Threshold Imaginary part

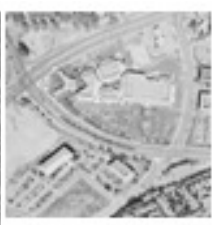

(f) Regeneration through (IZT)

Fig. 2. Forward $\mathrm{Z}$ transform followed by inverse transform over image

\subsection{Traditional $Z$ Transform}

In a specific case of Z-transform where we consider value of magnitude of $z(r=1)$, and the angular frequency $\omega €\{[0, \pi / 2, \pi, 3 \pi / 2]\}$. The forward Z-transformation for the vector $X$ (single mask of $2 \times 2$ ) is represented in figure $3(\mathrm{a})$, and the equation is given in eq (6). The elaborate form of eq (6) is shown in eq (7) where $C_{v}$ is the coefficient value.

\begin{tabular}{|l|l|}
\hline $\mathrm{X}_{00}$ & $\mathrm{X}_{01}$ \\
\hline $\mathrm{X}_{10}$ & $\mathrm{X}_{11}$ \\
\hline
\end{tabular}

(a) Single mask

\begin{tabular}{|l|l|}
\hline $\mathrm{R}_{00}$ & $\mathrm{R}_{01}$ \\
\hline $\mathrm{R}_{10}$ & $\mathrm{R}_{11}$ \\
\hline
\end{tabular}

(b) Real value after FZT

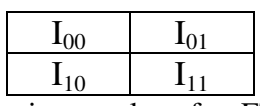

(c) Imaginary value after FZT

Fig. 2. Single mask representation of Image and its components after forward Z-Transformation (FZT).

$$
\begin{gathered}
X(z)=Z\{x[n]\}=\sum_{n=0}^{3} x[n] r^{-n}(\cos \omega+j \sin \omega)^{-n} \\
C_{v}=\left[x[0] r^{-0}(\cos \omega+j \sin \omega)^{-0}\right]+\left[x[1] r^{-1}(\cos \omega+\right. \\
\left.j \sin \omega)^{-1}\right]+\left[x[2] r^{-2}(\cos \omega+j \sin \omega)^{-2}\right]+ \\
{\left[x[3] r^{-3}(\cos \omega+j \sin \omega)^{-3}\right]}
\end{gathered}
$$

Different angular frequency $\omega €\{[0, \pi / 2, \pi, 3 \pi / 2]\}$ is taken and with $\mathrm{r}=1$ the complex coefficients values $C_{v}=R_{l m}+j I_{l m}$ are calculated by equation set eq $(8)$.

$$
R_{l m}+j I_{l m}=\sum_{k=0}^{3}\left[x[k] 1^{-k}(\cos \omega+\sin \omega)^{-k}\right]
$$

Here $\omega$ depends on the value of $1 \mathrm{~m}$.

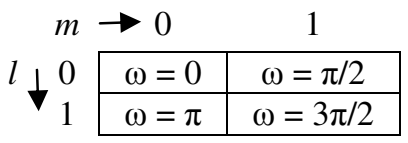

\subsection{Fast Z-Transformation}

Z-Transform can also be done with minimizing the computation and without the use of trigonometry, only by applying addition and subtraction. 


\section{Algorithm for forward Z-transformation}

Input: $\mathrm{X}_{00}, \mathrm{X}_{01}, \mathrm{X}_{10}$ and $\mathrm{X}_{11}$.

Output: $\mathrm{R}_{00}, \mathrm{R}_{01}, \mathrm{R}_{10}, \mathrm{R}_{11}, \mathrm{I}_{00}, \mathrm{I}_{01}, \mathrm{I}_{10}$, and $\mathrm{I}_{11}$.

Method: Perform arithmetic calculation for fast forward Z-transform.

Calculate $\mathrm{R}_{00}$ and $\mathrm{I}_{00}, \mathrm{R}_{10}$ and $\mathrm{I}_{10}, \mathrm{R}_{01}$ and $\mathrm{I}_{01}, \mathrm{R}_{11}$ and $\mathrm{I}_{11}$ by equation set (9), (10) and (11) respectively.

$$
\begin{gathered}
R_{00}=\sum_{i, j=0}^{1} X_{i j} \quad R_{10}=\sum_{i=0}^{1} X_{i 0}-\sum_{i=0}^{1} X_{i 1} \\
R_{01}+I_{01}=\sum_{i=0}^{1} X_{i i}-\sum_{i=0}^{1} X_{i i}, \\
\quad R_{01}-I_{01}=\sum_{i=0}^{1} X_{0 i}-\sum_{i=0}^{1} X_{1 i}
\end{gathered}
$$

Equation set 10 shows a relation as $a x+b y=c_{1}$ and $a x-b y=c_{2}$, where ' $a$ ' and ' $b$ ' are depend on the value of $\mathrm{r}$ used. Two unknown $\mathrm{x}$ and $\mathrm{y}$ needs to be calculated by two equations.

$$
\mathrm{R}_{11}=\mathrm{R}_{01} \text { and } \mathrm{I}_{11}=-\mathrm{I}_{01}
$$

\section{Algorithm for inverse Z-transformation}

In case of inverse transform the calculation will be followed through set of equations eq(12) and eq(13).

Input : $\mathrm{R}_{00}, \mathrm{R}_{01}, \mathrm{R}_{10}, \mathrm{R}_{11}, \mathrm{I}_{00}, \mathrm{I}_{01}, \mathrm{I}_{10}$, and $\mathrm{I}_{11}$.

Output: $\mathrm{X}_{00}, \mathrm{X}_{01}, \mathrm{X}_{10}$ and $\mathrm{X}_{11}$.

Method: Perform arithmetic calculation by equation set (12) and (13) for fast inverse Ztransformation.

Calculate $\mathrm{X}_{00}, \mathrm{X}_{01}$ by eq (12), and $\mathrm{X}_{10}$ and $\mathrm{X}_{11}$ by eq (13).

$$
\begin{gathered}
\mathrm{X}_{00}=\frac{1}{4} \sum_{\mathrm{i}, \mathrm{j}=0}^{1} \mathrm{R}_{\mathrm{ij}}+\mathrm{I}_{\mathrm{ij}} \quad \mathrm{X}_{01}=\frac{1}{4}\left\{\sum_{\mathrm{i}=0}^{1}\left(\mathrm{R}_{\mathrm{ii}}+\mathrm{I}_{\mathrm{ii}}\right)-\sum_{\mathrm{i}=0}^{1}\left(\mathrm{R}_{\mathrm{ii}}+\mathrm{I}_{\mathrm{ii}}\right)\right\} \\
\mathrm{X}_{10}=\frac{1}{4}\left\{\sum_{\mathrm{i}=0}^{1}\left(\mathrm{R}_{\mathrm{i} 0}+\mathrm{I}_{\mathrm{i} 0}\right)-\sum_{\mathrm{i}=0}^{1}\left(\mathrm{R}_{\mathrm{i} 1}+\mathrm{I}_{\mathrm{i} 1}\right)\right\} \mathrm{X}_{11}=\frac{1}{4}\left\{\sum_{\mathrm{i}=0}^{1}\left(\mathrm{R}_{0 \mathrm{i}}+\mathrm{I}_{0 \mathrm{i}}\right)-\sum_{\mathrm{i}=0}^{1}\left(\mathrm{R}_{1 \mathrm{i}}+\mathrm{I}_{1 \mathrm{i}}\right)\right\}
\end{gathered}
$$

\subsection{Bandwidth minimization/Transmission efficiency}

A 2 x 2 mask of spatial data on FZT generates two $2 \times 2$ matrixes containing real and imaginary frequency coefficient values, which means, information need to traverse after FZT will become double in terms of data. On analysis of eight subbands, four real and four imaginary valued, based on the temporary environment created for $\mathrm{z}$ transform, it can be clearly elucidate that at 
most six subbands are required at destination to regenerate the lossless image, due to the entire zero value imaginary part for lower frequency (LF) and vertical frequency (VF) (in this case due to $\omega)$. Two more subbands can reduce without any loss due to the complex conjugate pair of horizontal frequency (HF). Thus, in total, minimum four subbands are requirednout of eight to regenerate the original image without any loss as shown in figure 4.

\section{DISCUSSION ON RESULTS}

Benchmark (PGM) images [6] are taken to formulate results. IZEB is applied over gray scale images such as Baboon, Boat, Clock, Couple, Elaine, Jet, Space, Tank, Truck and Map with dimension $512 \times 512$, few results and shown in figure 4. Figure 4.a and 4.b represents the real and imaginary part, after forward Z-transformation respectively, and figure 4.c represents the four minimum subbands from real and imaginary parts that on inverse transform able to generate image back.

If we apply IZEB on cover images and calculate MSE and PSNR from reconstructed images at destination it is seen that MSE is zero and that of PSNR becomes $\infty$ (infinity) when four bands are transmitted. Other bands are computed at destination as given in table 1.

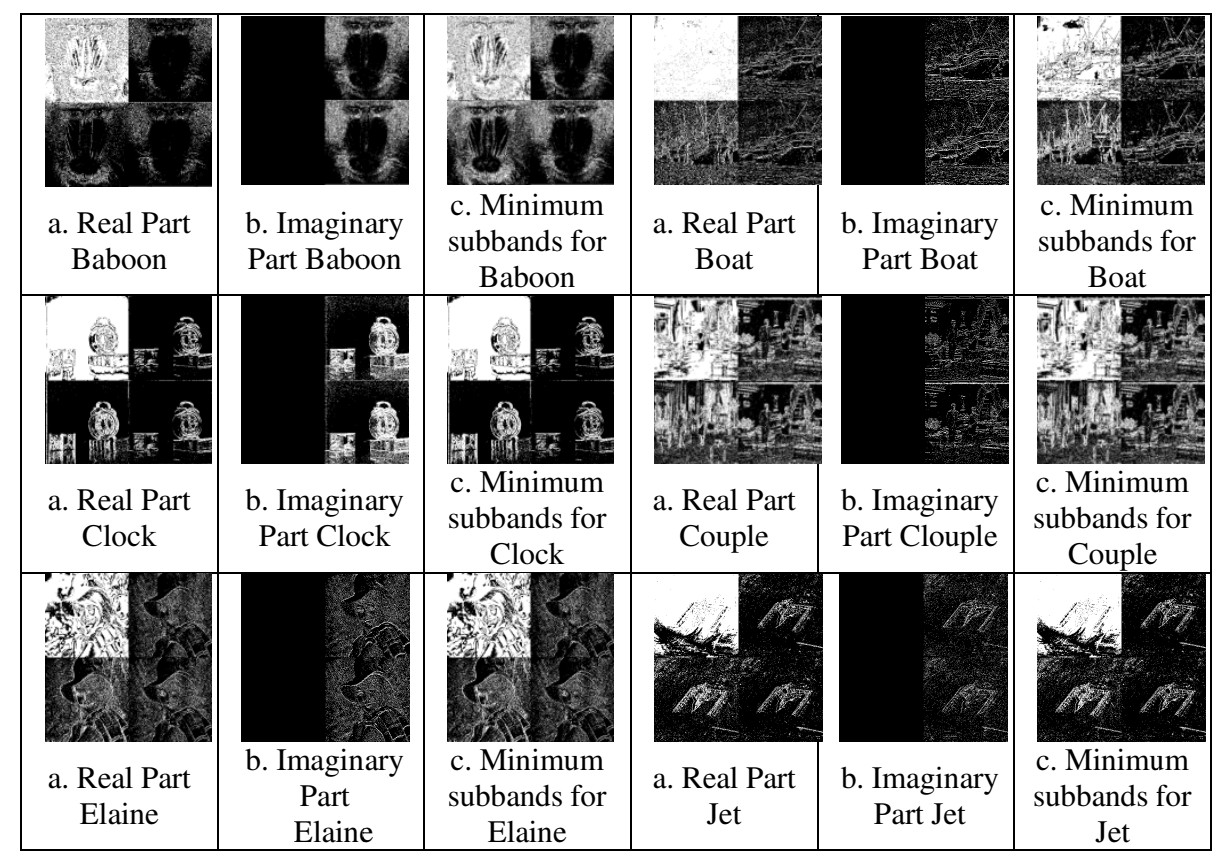

Fig. 3. Threshold image representing frequency coefficients after FZT.

Out of eight, four subbands are sufficient to regenerate lossless image at destination, thus minimizing the energy and the bandwidth to near about half. Further energy and bandwidth can be reduced further by reducing the subbands with little loss. Statistical calculations for amount of loss with different numbers of subbands sent to destination, calculated through IZT are shown in table 1 and in figure 5. It is clear from the table 1 that single band LF is enough to regenerate the image but LF needs maximum of 10 bit representation for every coefficient and rest of the bands are need 7 to 8 bit representations for coefficient. 
Table 1. Statistical Data of Loss with further reduced number of subbands

\begin{tabular}{|c|c|c|c|c|c|c|c|}
\hline \multirow[b]{2}{*}{$\begin{array}{l}\text { Cover } \\
\text { Image }\end{array}$} & \multirow[b]{2}{*}{$\begin{array}{c}\text { MSE/ } \\
\text { PSNR } \\
\text { (db) }\end{array}$} & \multicolumn{6}{|c|}{ Number and Name of Band used to reconstruct through IZT } \\
\hline & & 1। LF & $\begin{array}{c}\text { 2| LF \& } \\
\text { HF }\end{array}$ & $\begin{array}{l}\text { 2) LF \& } \\
\text { VF }\end{array}$ & $\begin{array}{c}\text { 3l LF, } \\
\text { HF \& VF }\end{array}$ & $\begin{array}{l}3 \mid \mathrm{LF} \text {, } \\
\mathrm{HF} \& \text { } \\
\text { imgHF }\end{array}$ & $\begin{array}{c}4 \mid \\
\text { LF,HF, } \\
\text { VF \& } \\
\text { imgHF }\end{array}$ \\
\hline Clock & MSE & 26.827541 & 29.483864 & 41.385128 & 44.007004 & 27.010376 & 0.000 \\
\hline .pgm & $\begin{array}{l}\text { PSNR } \\
\end{array}$ & 33.844995 & 33.434960 & 31.962361 & 31.695586 & 33.815497 & $\infty$ \\
\hline
\end{tabular}

\begin{tabular}{|c|c|c|c|c|c|c|c|}
\hline Elaine & MSE & 43.429588 & 58.092613 & 62.663673 & 77.373775 & 47.226242 & 0.000 \\
\cline { 2 - 9 } .pgm & PSNR & 31.752946 & 30.489594 & 30.160645 & 29.244866 & 31.388970 & $\infty$ \\
\hline $\begin{array}{c}\text { Space } \\
\text {.pgm }\end{array}$ & MSE & 12.659260 & 14.525913 & 20.651455 & 22.501396 & 13.147259 & 0.000 \\
\cline { 2 - 9 } & PSNR & 37.106720 & 36.509369 & 34.981297 & 34.608709 & 36.942452 & $\infty$ \\
\hline $\begin{array}{c}\text { Tank. } \\
\text { pgm }\end{array}$ & MSE & 39.988754 & 53.691200 & 53.740608 & 67.420712 & 43.637718 & 0.000 \\
\cline { 2 - 9 } & PSNR & 32.111425 & 30.831772 & 30.827778 & 29.842870 & 31.732183 & $\infty$ \\
\hline $\begin{array}{c}\text { Truck } \\
\text {.pgm }\end{array}$ & MSE & 39.040760 & 56.560173 & 48.327442 & 66.218987 & 44.382500 & 0.000 \\
\cline { 2 - 9 } & PSNR & 32.215621 & 30.605696 & 31.288866 & 29.920978 & 31.658686 & $\infty$ \\
\hline
\end{tabular}

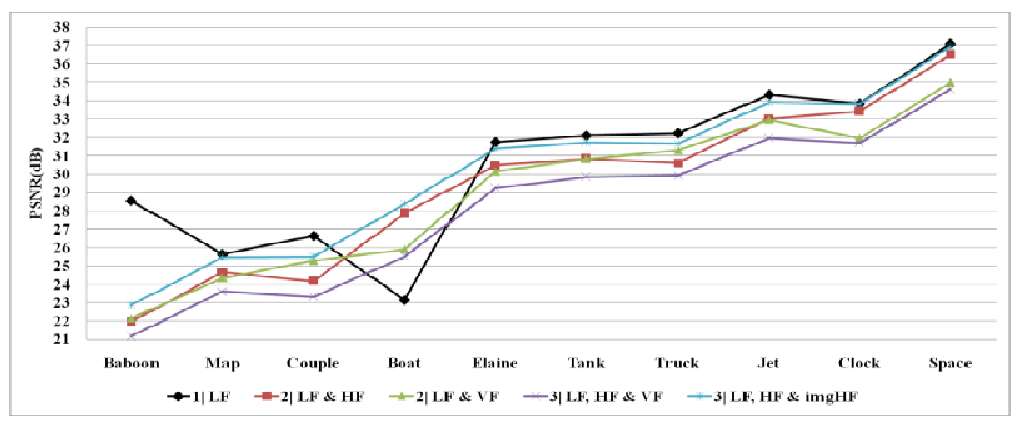

Fig. 4. Comparison in terms of Energy/bandwidth verses PSNR (Table 1)

In case of original image of $512 \times 512$ in dimension, to send spatial data, transmission medium need to send $512 * 512 * 8=2097152$ bits. FZT complex coefficients take $512 * 512 * 2 * 8=4194304$ bits. For LF band $256 * 256 * 10=655360$ bits required, for rest of the bands 8 bit per coefficient is required. All the computational results are given in table II and visual interpretation of the same is shown in figure 6.

Table 2. Number of bits nedded to be send for transmission of $512 \times 512$ dimension image

\begin{tabular}{c|c|c}
\hline Slno & Number \& name of subbands & Number of bits \\
\hline 1. & Original Image in spatial domain & 2097152 \\
2. & Complete Z-transform coefficient & 4194304 \\
3. & 4l LF,HF, VF \& imgHF & 2228224 \\
4. & 3I LF, HF \& VF & 1703936 \\
5. & 2I LF \& HF & 1179648 \\
6. & 2 LF \& VF & 1179648 \\
7. & 1 LF & 655360 \\
\hline
\end{tabular}




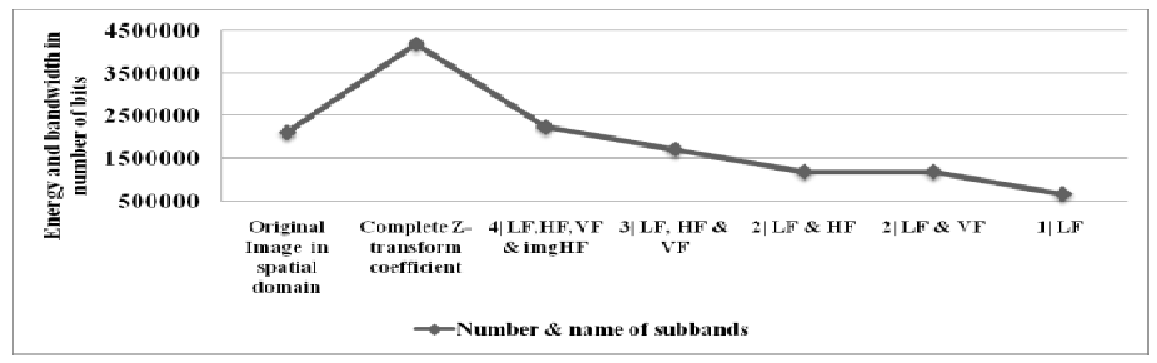

Fig. 5. Graphical representation of energy/bandwidth in bits with various band(s)

\section{CONCLUSiOnS}

The issue of image coding with minimum calculation and less complexity is achieved in this proposed work. IZEB also emphasis the subband minimization technique, out of eight subbands only four is enough to regenerate the image without loss this lower the energy consumption and the bandwidth too.

\section{REFERENCES}

[1] Ghoshal, Nabin, and Mandal, Jyotsna Kumar, "A Novel Technique for Image Authentication in Frequency Domain Using Discrete Fourier Transformation Technique (IAFDDFTT)". Malaysian Journal of Computer Science, Vol 21 (No. 1). pp. 24-32. ISSN 0127-9084, University of Malaya, Kuala Lumpur, Malyasia, 2008.

[2] Madhumita Sengupta, J. K. Mandal, "Authentication of Images through Non Convoluted DCT (AINCDCT)", first International Conference on Communication and Industrial Application (ICCIA 2011), Dec, 26-28, 2011, IEEE, pp- 1-4, DOI: 10.1109/ICCIndA.2011.6146672.

[3] Madhumita Sengupta, J. K. Mandal, Nabin Ghoshal, "An authetication technique in frequency domain through wavelet transform (ATFDWT), Advances in Modelling Signal Processing and Pattern Recognition(AMSE), vol-54, Issue 2, 20011.

[4] J. K. Mandal, Madhumita Sengupta, "Steganographic Technique Based on Minimum Deviation of Fidelity (STMDF)", IEEE, Second International Conference on Emerging Applications of Information Technology (EAIT 2011), Print ISBN: 978-1-4244-9683-9, DOI: 10.1109/EAIT.2011.24, Issue Date: 19-20 Feb. 2011 pp- 298 - 301.

[5] J. K. Mandal, Madhumita Sengupta, "Authentication /Secret Message Transformation Through Wavelet Transform based Subband Image Coding (WTSIC)", IEEE, ISED- 2010, pp 225-229, ISBN 978-0-7695-4294-2, Bhubaneswar, India, Print ISBN: 978-1-4244-8979-4, Dec, 20th -22nd, 2010, DOI 10.1109/ISED.2010.50.

[6] Allan G. Weber, The USC-SIPI Image Database: Version 5, Original release: October 1997, Signal and Image Processing Institute, University of Southern California, Department of Electrical Engineering. http://sipi.usc.edu/database/ (accessed on 25th January, 2012).

[7] Kutter M , Petitcolas F A P. A fair benchmark for image watermarking systems, Electronic Imaging 99. Security and Watermarking of Multimedia Contents. vol. 3657. Sans Jose, CA, USA. January 1999. The International Society for Optical Engineering. pp 226-239,1999. 